\title{
A note on the Chermak-Delgado lattice of a finite group
}

\author{
Marius Tărnăuceanu \\ December 9, 2016
}

\begin{abstract}
In this note we describe the structure of finite groups $G$ whose Chermak-Delgado lattice is the interval $[G / Z(G)]=\{H \in L(G) \mid$ $Z(G) \leq H \leq G\}$.
\end{abstract}

MSC2000 : Primary 20D30; Secondary 20D15, 20E34.

Key words: Chermak-Delgado measure, Chermak-Delgado lattice, centralizer lattice, subgroup lattice.

\section{Introduction}

Let $G$ be a finite group and $L(G)$ be the subgroup lattice of $G$. The ChermakDelgado measure of a subgroup $H$ of $G$ is defined by

$$
m_{G}(H)=|H|\left|C_{G}(H)\right| \text {. }
$$

Let

$$
m(G)=\max \left\{m_{G}(H) \mid H \leq G\right\} \text { and } \mathcal{C D}(G)=\left\{H \leq G \mid m_{G}(H)=m(G)\right\} .
$$

Then the set $\mathcal{C D}(G)$ forms a modular self-dual sublattice of $L(G)$, which is called the Chermak-Delgado lattice of $G$. It was first introduced by Chermak and Delgado [5], and revisited by Isaacs [7]. In the last years there has been a growing interest in understanding this lattice, especially for $p$-groups (see e.g. [1, 2, 3, 10]). The study can be naturally extended to nilpotent groups, since by 2] the Chermak-Delgado lattice of a direct product of finite 
groups decomposes as the direct product of the Chermak-Delgado lattices of the factors. Recall also that if $H \in \mathcal{C D}(G)$, then $C_{G}(H) \in \mathcal{C D}(G)$ and $C_{G}\left(C_{G}(H)\right)=H$. This implies that $\mathcal{C D}(G)$ is contained in the centralizer lattice $\mathfrak{C}(G)$ of $G$.

We remark that $\mathcal{C D}(G)=[G / Z(G)]$ for many finite groups $G$, such us $D_{8}, Q_{8}$, any abelian group, ... and so on. Thus, the study of finite groups satisfying this property is very natural. It is the goal of the current note. Our main result is stated as follows.

Theorem 1. Let $G$ be a finite group. Then $\mathcal{C D}(G)=[G / Z(G)]$ if and only if $G=G_{1} \times \cdots \times G_{r} \times A$, where

$$
\operatorname{gcd}\left(\left|G_{i}\right|,\left|G_{j}\right|\right)=1=\operatorname{gcd}\left(\left|G_{i}\right|,|A|\right) \text { for all } i \neq j
$$

$A$ is an abelian group, and every $G_{i}$ is a p-group satisfying

$$
\left[G_{i} / Z\left(G_{i}\right)\right] \text { is modular and } G_{i}^{\prime} \text { is cyclic. }
$$

Note that the conditions (1) are equivalent with the conditions

$$
G_{i}^{\prime}=\langle a\rangle \text { is cyclic and }\left[\langle a\rangle, G_{i}\right] \leq\left\langle a^{4}\right\rangle
$$

by Theorem 9.3 .19 of [9] (see also [4, 8]).

The following corollary is an immediate consequence of Theorem 1.

Corollary 2. Every finite group $G$ satisfying $\mathcal{C D}(G)=[G / Z(G)]$ is nilpotent.

By Corollary 9.3.18 of [9] (see also [6]), we know that there are finite groups in which every subgroup is a centralizer. Theorem 1 shows that a similar result does not hold for the Chermak-Delgado lattice.

Corollary 3. There is no finite non-trivial group $G$ such that $\mathcal{C D}(G)=$ $L(G)$.

Also, Theorem 1 shows that the property $\mathcal{C D}(G)=[G / Z(G)]$ is inherited by subgroups.

Corollary 4. If $G$ is a finite group satisfying $\mathcal{C D}(G)=[G / Z(G)]$ and $H$ is a subgroup of $G$, then $\mathcal{C D}(H)=[H / Z(H)]$. 
Observe that if for a finite group $G$ we have $\mathcal{C D}(G)=[G / Z(G)]$, then $\mathfrak{C}(G)=[G / Z(G)]$ and so $\mathfrak{C}(G)=\mathcal{C D}(G)$ is a modular lattice. Moreover, its length $l$ must be even by Lemma 9.3 .10 of [9]. Elementary examples of such groups are all abelian groups for $l=0$, and $D_{8}, Q_{8}$ for $l=2$. A more general example is the following.

Example. Let $G$ be an extra-special group $G$ of order $p^{2 n+1}$. Then

$$
G / Z(G) \cong \mathbb{Z}_{p}^{2 n} \text { is modular and } G^{\prime} \cong \mathbb{Z}_{p} \text { is cyclic, }
$$

and therefore $\mathcal{C D}(G)=[G / Z(G)]$ is a modular lattice of length $2 n$.

Finally, we indicate a natural open problem concerning the above study.

Open problem. Describe the structure of finite groups $G$ such that $\mathcal{C D}(G)$ is an interval (not necessarily $[G / Z(G)]$ ) of $L(G)$.

\section{Proof of the main result}

We start by proving an auxiliary result.

Lemma 5. Let $G$ be a finite p-group. Then $\mathcal{C D}(G)=[G / Z(G)]$ if and only if $[G / Z(G)]$ is modular and $G^{\prime}$ is cyclic.

Proof. If $\mathcal{C D}(G)=[G / Z(G)]$, then $\mathfrak{C}(G)=[G / Z(G)]$ and so $[G / Z(G)]$ is modular and $G^{\prime}$ is cyclic by Theorem 9.3 .19 of [9].

Conversely, if $[G / Z(G)]$ is modular and $G^{\prime}$ is cyclic, then $\mathfrak{C}(G)=[G / Z(G)]$. By Lemma 4 of [4] we infer that

$$
m_{G}(H)=|H|\left|C_{G}(H)\right|=|G||Z(G)|, \forall H \in[G / Z(G)],
$$

that is all subgroups in $[G / Z(G)]$ have the same Chermak-Delgado measure. This shows that $\mathcal{C D}(G)=[G / Z(G)]$, as desired.

Remark. Let $G$ be a non-abelian $p$-group of order $p^{n}$ satisfying $\mathcal{C D}(G)=$ $[G / Z(G)]$. If $G$ contains an abelian subgroup $M$ of order $p^{n-1}$ (as it happens for $D_{8}$ and $\left.Q_{8}\right)$, then $(G: Z(G))=p^{2}$.

Indeed, we have $M \subseteq C_{G}(M)$ because $M$ is abelian and thus

$$
|G||Z(G)|=m(G)=|M|\left|C_{G}(M)\right| \geq|M|^{2} .
$$


One obtains

$$
p^{2} \leq(G: Z(G)) \leq(G: M)^{2}=p^{2},
$$

that is $(G: Z(G))=p^{2}$.

We are now able to prove our main theorem.

Proof of Theorem 1. Assume first that $G=G_{1} \times \cdots \times G_{r} \times A$, where $G_{i}$, $i=1, \ldots, r$, and $A$ satisfy the conditions in Theorem 1 . Then

$$
\mathcal{C D}\left(G_{i}\right)=\left[G_{i} / Z\left(G_{i}\right)\right], \forall i=1, \ldots, r
$$

by Lemma 5. It follows that

$$
\begin{aligned}
\mathcal{C D}(G) & =\mathcal{C D}\left(G_{1}\right) \times \cdots \times \mathcal{C D}\left(G_{r}\right) \times\{A\} \\
& =\left[G_{1} / Z\left(G_{1}\right)\right] \times \cdots \times\left[G_{r} / Z\left(G_{r}\right)\right] \times\{A\} \\
& =[G / Z(G)] .
\end{aligned}
$$

Conversely, assume that $\mathcal{C D}(G)=[G / Z(G)]$. Since $\mathcal{C D}(G) \subseteq \mathfrak{C}(G)$, we infer that $\mathfrak{C}(G)=[G / Z(G)]$. Then Theorem 9.3.17 of [9] implies that $G=G_{1} \times \cdots \times G_{r} \times A$, where

$$
\operatorname{gcd}\left(\left|G_{i}\right|,\left|G_{j}\right|\right)=1=\operatorname{gcd}\left(\left|G_{i}\right|,|A|\right) \text { for all } i \neq j,
$$

$A$ is an abelian group, and every $G_{i}$ is either a $\{p, q\}$-group with $\left|G_{i} / Z\left(G_{i}\right)\right|=$ $p q$ or a $p$-group satisfying $\mathfrak{C}\left(G_{i}\right)=\left[G_{i} / Z\left(G_{i}\right)\right], p$ and $q$ primes. Clearly, this leads to

$$
\mathcal{C D}(G)=\mathcal{C D}\left(G_{1}\right) \times \cdots \times \mathcal{C D}\left(G_{r}\right) \times\{A\}
$$

and

$$
[G / Z(G)]=\left[G_{1} / Z\left(G_{1}\right)\right] \times \cdots \times\left[G_{r} / Z\left(G_{r}\right)\right]
$$

implying that

$$
\mathcal{C D}\left(G_{i}\right)=\left[G_{i} / Z\left(G_{i}\right)\right], \forall i=1, \ldots, r .
$$

If $G_{i}$ would be a $\{p, q\}$-group with $\left|G_{i} / Z\left(G_{i}\right)\right|=p q$ and $p<q$, then $\mathcal{C D}\left(G_{i}\right)$ would consists only of the unique subgroup of index $p$ contained in $\left[G_{i} / Z\left(G_{i}\right)\right]$, a contradiction. Consequently, $G_{i}$ is a $p$-group. On the other hand, it satisfies the conditions (1) by Theorem 9.3.19 of [9]. This completes the proof. 


\section{References}

[1] L. An, J.P. Brennan, H. Qu and E. Wilcox, Chermak-Delgado lattice extension theorems, Comm. Algebra 43 (2015), 2201-2213.

[2] B. Brewster and E. Wilcox, Some groups with computable ChermakDelgado lattices, Bull. Aus. Math. Soc. 86 (2012), 29-40.

[3] B. Brewster, P. Hauck and E. Wilcox, Groups whose Chermak-Delgado lattice is a chain, J. Group Theory 17 (2014), 253-279.

[4] A. Cheng, On finite p-groups with cyclic commutator subgroup, Arch. Math. 39 (1982), 295-298.

[5] A. Chermak and A. Delgado, A measuring argument for finite groups, Proc. AMS 107 (1989), 907-914.

[6] W. Gaschütz, Gruppen, deren sämtliche Untergruppen Zentralisatoren sind, Arch. Math. 6 (1954), 5-8.

[7] I.M. Isaacs, Finite group theory, Amer. Math. Soc., Providence, R.I., 2008.

[8] M. Reuther, Endliche Gruppen, in denen alle das Zentrum enthaltenden Untergruppen Zentralisatoren sind, Arch. Math. 29, 45-54.

[9] R. Schmidt, Subgroup lattices of groups, de Gruyter Expositions in Mathematics 14, de Gruyter, Berlin, 1994.

[10] E. Wilcox, Exploring the Chermak-Delgado lattice, Math. Magazine 89 (2016), 38-44.

Marius Tărnăuceanu

Faculty of Mathematics

"Al.I. Cuza" University

Iaşi, Romania

e-mail: tarnauc@uaic.ro 